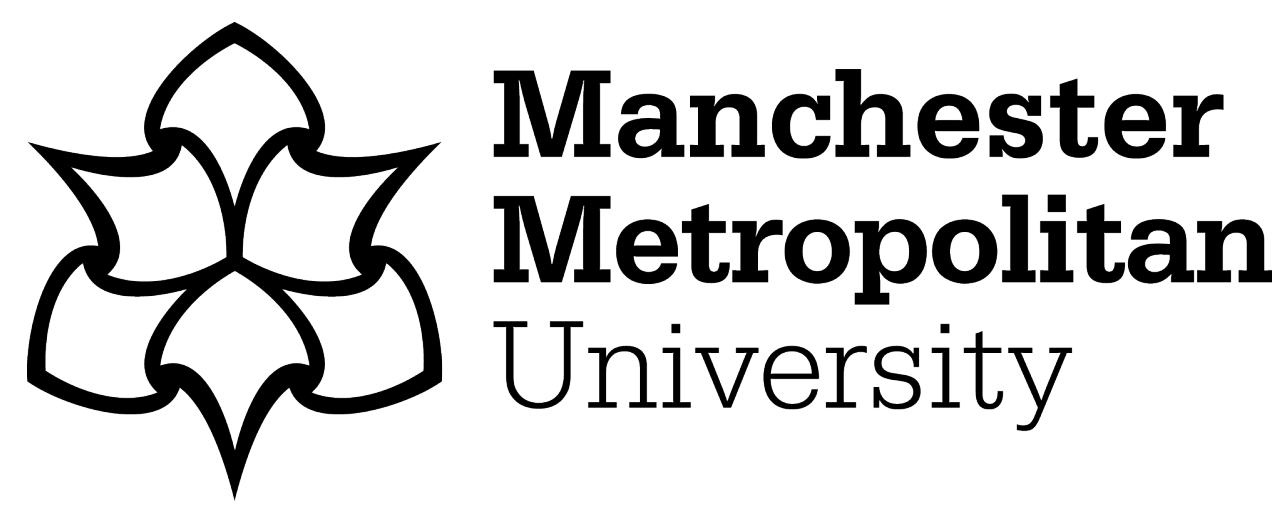

Tavana, M, Kazemi, MR, Vafadarnikjoo, A ORCID logoORCID: https://orcid.org/0000-0003-2147-6043 and Mobin, M (2016) An artificial immune algorithm for ergonomic product classification using anthropometric measurements. Measurement, 94. pp. 621-629. ISSN 0263-2241

Downloaded from: https://e-space.mmu.ac.uk/627673/

Version: Accepted Version

Publisher: Elsevier

DOI: https://doi.org/10.1016/j.measurement.2016.09.007

Usage rights: Creative Commons: Attribution-Noncommercial-No Derivative Works 4.0

Please cite the published version 


\title{
An Artificial Immune Algorithm for Ergonomic Product Classification Using Anthropometric Measurements
}

\author{
Madjid Tavana $a^{a, b}, *$ \\ aBusiness Systems and Analytics Department \\ Distinguished Chair of Business Analytics \\ La Salle University, Philadelphia, PA19141, USA \\ E-mail: tavana@lasalle.edu \\ Web: http://tavana.us/ \\ ${ }^{\mathbf{b}}$ Business Information Systems Department \\ Faculty of Business Administration and Economics \\ University of Paderborn, D-33098 Paderborn, Germany \\ Mohammad Reza Kazemi ${ }^{\mathrm{c}}$ \\ 'Industrial Engineering Department \\ Mazandaran University of Science and Technology, Babol, Iran \\ Amin Vafadarnikjoo ${ }^{d}$ \\ ${ }^{\mathbf{d}}$ Management and Accounting Department \\ Allameh Tabataba'i University, Tehran, Iran \\ Mohammadsadegh Mobin ${ }^{\mathrm{e}}$ \\ eIndustrial Engineering and Engineering Management Department \\ Western New England University, Springfield, MA 01119, USA
}

DOI: https://doi.org/10.1016/i.measurement.2016.09.007

*Corresponding author at: Distinguished Chair of Business Systems and Analytics, La Salle University, Philadelphia, PA 19141, United States. Tel.: +1 215951 1129; fax: +1 2672952854. 


\title{
An Artificial Immune Algorithm for Ergonomic Product Classification Using Anthropometric Measurements
}

\begin{abstract}
Product classification using anthropometric measurements leads to ergonomic product design and user satisfaction. We propose an effective artificial immune algorithm (AIA) to classify ergonomic products with multi-criteria anthropometric measurements and tune the AIA parameters with a full factorial experimental design approach. We demonstrate the applicability and efficacy of the proposed algorithm by considering the anthropometric measurements of the hand, developing an ergonomic computer mouse, and classifying consumers into three categories. The resulting classifications are compared with expert opinions to facilitate the conformity of the computer mouse to user requirements.
\end{abstract}

Keywords: ergonomic product classification; anthropometric measurements; artificial immune algorithm; ergonomic product design; meta-heuristic. 


\section{Introduction}

Ergonomics is the science of fitting a job to a worker and a product to a user (Pheasant, 2003). One of the basic challenges facing ergonomics is preventive health care. The goal of averting occupationally-generated musculoskeletal disorders and related illnesses can be attained by a suitable design of workplace and product (Schaub et al., 1997). Anthropometry is a field of science that deals with body measurements. It is associated with the physical characteristics of humans in different situations, especially with measurements of body size, shape, strength, and working capacity (Pheasant, 2003; Nowak, 1996). In the related literature, anthropometry is mentioned as a very consequential division of ergonomics because misalliances between human anthropometric dimensions and equipment dimensions may lead to inconvenience, plunging productivity, accidents, biomechanical stress, fatigue, injuries, and cumulative traumas (Mandahawi et al., 2008; Kar et al., 2003; Okunribido 2000). Jung et al. (2000) expressed that operator's anthropometry, task geometry, and design factors would be central components for an optimal ergonomic product design. There are a huge amount of hand tool injuries each year worldwide which are partly related to worker-tool mismatches (Aghazadeh and Mital, 1987). Pheasant (2003) revealed that in order to design ergonomic products, as well as, to have compatibility between products and users, many factors should be considered. These factors include anthropometric characteristics of users, ways in which these characteristics impose constraints upon ergonomic product design process, and eventually, criteria that define a successful match between products and users. Hsu (2009) developed accurate industrial standards and utilized an accurate girth ratio approach which is used to ascertain figure types enabling the production process in apparel manufacturing to be based on anthropometric data.

There are two types of anthropometric information including structural and functional information. Structural or static anthropometric information is linked to a body's measure or size in a fixed structural position and it is generally measured by marked anatomic points in a certain position. The second type of anthropometric information, which includes measures of access limits and is measured in practical conditions, is functional or dynamic anthropometric information (Pheasant, 2003). Anthropometric measurements or morphologies of human body parts have been explored in a myriad of studies. Lackovic et al. (2000) presented a system for the purpose of force measurement in the legs and crutches based on a quantitative gait analysis of 
orthopaedic patients. Kieckhoefer et al. (2006) investigated ear print formation considering the morphology of an auricle and developed the appropriate optical equipment for this purpose.

This article is primarily concerned with the anthropometric side of ergonomics, which considers matching the physical form and dimensions of a product to those of its users and, likewise, matching physical demands of a working task to the capacities of a workforce. In most real-world design problems, the concentration is on the users of products, while the structural or static anthropometric information is considered in this paper.

Humans in a population vary based on the proportion of their bodies. It has been shown in the literature that despite the goal of having $95 \%$ of the user population in the ergonomic product design interval, in fact $25 \%$ of the population will fall out of the design limit which means that only $75 \%$ of the population will be in the design limit (Pheasant, 2003; Bittner et al., 1975). This result indicates the significance of taking into account anthropometric data in the real ergonomic product design, and consequently the probable effects of the ergonomic product design on humans. For instance, Lacko et al. (2017) utilized 3D anthropometric data as a feasible design method for brain-computer interfacing (BCI) headgear to create a one-size BCI headset prototype which fits all individuals. Chen et al. (2016) have taken into account ergonomic considerations in order to design a wearable device for frozen shoulder rehabilitation. Garcia and Garcia-Mendoza (2015) proposed an approach based on optimal ergonomic specifications in order to design mobility devices. Vincent et al. (2014) investigated the constraints that influence the medical device design and development process. Wu et al. (2009) applied a user-centered design approach to develop a hair washer for disabled people for use in normal postures. Martin et al. (2008) reviewed the literature on methods for evaluating user requirements in ergonomics as well as examining factors that affect user requirements for medical device design.

Meanwhile, the classification of items can assist designers with conformity of tools/products and users/clients. Item classification has a variety of applications (e.g., clothing, desks, chairs, and other instruments humans utilize) which leads to a more comfortable user experience. Classification of technologies and products/services should facilitate the decision making process regarding realization of the system functionalities (Pääkkönen and Pakkala, 2015). Classification in its most straightforward form attempts to assign some unknown objects into a known class of objects. Customarily, these classes are stereotypes which are hierarchically 
organized. (Clancey, 1984).

The classification problem has been applied in varied realms such as computer science, engineering, image processing, and data mining (Zhu and Guan, 2004). Classification also has empirical applications in performing medical diagnosis (Stefanowski and Slowinski, 1998; Tsumoto, 1998; Belacel, 2000; Michalowski et al., 2001), fault detection (Li et al., 2013; Illias and Chai, 2016), assignment of personnel into proper occupational categories based on their qualifications (Rulon et al., 1967; Gochet et al., 1997), and customer satisfaction evaluation and characteristics analysis of different groups of customers (Dutka, 1995; Siskos et al., 1998). Discerned human related emotions should be considered as pivotal components in the ergonomic product design process of a product. However, customers' affective needs are arduous to perceive. Therefore, product designers often incorrectly comprehend what customers really want most of the time. Bahn et al. (2009) proposed a design framework by considering critical affective features of customers on products, and systematically, incorporated these types of features into ergonomic product design attributes. This broad span of practical applications of classification motivates researchers to develop various methodologies for the classification problem. Nagarajan and Balasubramanie (2008) utilized a neural-based classifier approach for object classification which detects car objects in the midst of a cluttered background. Ghazali et al. (2008) introduced the Scale Invariant Feature Transform (SIFT) technique to overcome the problem of weed classification in a hands-on application of image processing. Neural networks (Anand et al., 1995; Lu and Ito, 1999; Guan and Li, 2003), evolutionary algorithms (Corcoran and Sen, 1994; Brameier and Banzhaf, 2001; Falco et al., 2002), and fuzzy logic (Ishibuchi et al., 1999; Setnes and Roubos, 2000) are some other soft computing approaches that have been extensively applied to adaptively evolve solutions for classification issues. The Genetic Algorithm (GA) method has attracted much attention and has become one of the most popular techniques for classification (Merelo et al., 2001). However, GA suffers from a number of weaknesses, particularly in larger scale real world classification problems, such as encountering inefficiency in searching a large space, finding it difficult to break internal interference of training data, and getting trapped in local optima (Zhu and Guan, 2004).

Producers need to draw the attention of more consumers in the present-day competitive markets, and on the other hand, consumers' views are vital motivating factors for product development and obtaining profit in the market place. Hence, producers are required to design 
products that satisfy the requirements of the majority of people and also provide comfort when they are used based on ergonomic criteria (Liu, 2008). However, only very limited researches have been carried out analyzing hand sizing, which is essential for the design of hand tools. Kwon et al. (2009) developed a glove sizing system that was based on three hand dimensions (length, circumference, and breadth). Pekelney and Chu (1995) described the ergonomic design criteria and product attributes of a computer mouse device. Hsiao et al. (2015) proposed an improved sizing scheme for fire-fighting gloves that appropriately fits the US firemen population. In another study, Liu and Fan (2014) considered four anthropometric measurements of the hand (width and length of hand, palm, and index) in their study to design an ergonomic computer mouse for clients with wrist splints.

Thus, the significance of a technique which considers various anthropometric criteria in designing an ergonomic product-computer mouse with all real-world limitations is completely obvious. To tackle the above mentioned difficulties, a meta-heuristic method, called the artificial immune algorithm (AIA), is applied. Its name reflects the fact that it is driven by the natural immune system of the human body which is an origin of inventiveness for resolving optimization problems. There are other methods such as principal component analysis (PCA) and the discriminant analysis technique which may be utilized in this case, but the AIA has the capability of learning and mimicking the categorizing ability of a human expert in the field. An AIA imitates the learning ability carried out by the natural immune system (Kalinli and Karaboga, 2005).

In this paper, the classification of an ergonomic computer mouse is studied considering multi-criteria anthropometric measurements using an AIA. A problem of user classification into small (S), medium (M), and large (L) categories for validation is considered. Here, the artificial immune system algorithm is applied to learn the weights of the criteria along with SM (SmallMedium) and ML (Medium-Large) cut-off points from pre-classified items based on the research by Zandie et al. (2013). Anthropometric measurements of five areas of the hand are considered in this study. The classifications resulting from the AIA is compared with an expert opinion, one who has the full authority and expertise to classify items on the basis of the mentioned anthropometric data. The main aim of this study is to provide a method to facilitate the conformity of products to users according to anthropometric measurements.

The rest of this article is organized as follows. The problem description is presented in 
Section 2. The general mechanism of the artificial immune system and the proposed AIA are elaborated in Section 3. The computational results and parameters tuning of the AIA are presented in Section 4. Finally, the conclusion highlights are presented in Section 5.

\section{Problem Description}

Wrist pain is a frequent ailment and many kinds of wrist pains are caused by sudden injuries which lead to sprains or fractures. Wrist pain can be brought about by more chronic problems as well, such as repetitive stress, arthritis and carpal tunnel syndrome as well. When hand tools are bigger or smaller than the hand size, long-term use can cause wrist pain. Liu and Fan (2014) considered four anthropometric measurements of the hand (width and length, palm, and index) in their study to examine the effects of cutaneous feedback and hump position on effective use of the computer mouse with a splint. Furthermore, Kwon et al. (2009) selected three pivotal hand dimensions (length, circumferences, and breadth) to design gloves for candidates.

The way the computer mouse and keyboard are used probably poses a risk of carpal tunnel syndrome and repetitive and sustained loading of the small muscles of the hand. It is highlighted in the literature that a vertical mouse and ergonomic mouse pads change wrist position; however, they do not alleviate carpal tunnel pressure in patients with carpal tunnel syndrome (Rempel et al., 1997; Rempel et al., 1998; Fung et al., 2007; Keir et al., 1999). It is reported that mouse users adopt working postures of wrist extension, pronation, and ulnar deviation (Karlqvist et al., 1994; Fernström and Ericson, 1997; Cook and Kothiyal, 1998; Burgess-Limerick et al., 1999). Liu and Fan (2014) carried out a computer task by thirty participants utilizing two forms of computer mouses (front hump and rear hump) and two types of wrist splints (dorsal and volar) and measured the movement times and satisfaction scores.

The computer mouse is one of the most functional devices that can influence the wrists of its users negatively. A study of the literature and industrial practices show that anthropometric measures of five important areas of the hand have been used to classify computer mouse users into small, medium and large categories and ultimately design an ergonomic product. The hand areas, which are presented in Figure 1, include: A: wrist width; B: wrist length; C: palm width; D: middle finger length; and E: index finger length.

\section{Insert Figure 1 Here}

The seventy measured samples (both right and left hands) are selected from 35 individuals. Seventy hand dimensions were selected based upon the 1988 US Army hand 
anthropometric survey report (Greiner, 1991), after which they are categorized into three dimensional groups (length, circumference, and breadth) (Kwon et al., 2009).

\section{Artificial Immune Algorithm}

In this research, an artificial immune system algorithm is developed to categorize computer mouse users based on their hand size and finally produce computer mouses which match effectively with anthropometric measurements of users' hands. The artificial immune system and its algorithm are presented as follows.

An AIA imitates a learning technique performed by the natural immune system (Kalinli and Karaboga, 2005) and is one of the methodologies inspired from biological systems. The natural immune system is an intricate adaptive pattern-recognition system that guards the body from bacteria or viruses (pathogens). It can classify all cells, i.e., or molecules, within the body as either those are members of its own kind (self-cell) or those that have a foreign origin (non self-cell) (Dasgupta, 2002).

The human body is under continuous attack from bacteria or viruses, or disease-carrying parasites. Immune system consists of two layers of protection, non-specific and specific defense. The first defensive line is to prevent pathogens from entering the body, called non-specific defense, which includes skin and mucous membranes. The specific defense is activated when these micro-organisms get past the non-specific defense and invade the body. Two activities must occur to make the immune system work properly: First, the body must recognize that it has been invaded, either by pathogens or toxins. Second, before invaders destroy many body tissue cells, the immune response must be activated swiftly. In order to respond effectively, several conditions including the proper interaction of non-specific and specific defense must be in place. When non-specific defense cannot destroy micro-organisms and is proven to be ineffective, specific defense goes into action.

According to the information processing perspective, an immune system is an exceptional adaptive system and can shed light on many significant aspects in the realm of computation (Frank et al., 1996; Dasgupta and Attoh-Okine, 1997). The immune system provides a way to solve optimization problems more effectively. When the immune system combines with evolutionary algorithms, it can promote the search capability during the evolutionary process (Jiao and Wang, 2000). Artificial immune systems and their applications have been extensively investigated in the literature (Komaki et al., 2016; Souza et al., 2016; Chandrasekar and Suresh 
Kumar, 2016; Miyamoto et al., 2004; Watkins et al., 2004; Xiaoping et al., 2003; Kalinli and Karaboga, 2003; Hong et al., 2000; Kalinli and Karaboga, 2005; Chen et al. 2013).

\subsection{Implementation of Immune System Algorithm}

The artificial immune system algorithm is applied to learn the weight of criteria along with SM (Small-Medium) and ML (Medium-Large) cut-off points from pre-classified items based on the research by Zandieh et al. (2013). A detailed description of the AIA is presented as follows:

\section{Encoding Mechanism}

In a classification problem a set of antibodies representing one class or category, is used as a solution. These antibodies are randomly generated by computerized procedures. Antibodies will be replaced by others or random mutation will occur, at a time when a set of antibodies do not have high fitness. Replacement happens based on the replacement probability value. High fitness antibodies with high resemblance to each other result in a lack of uniformity of antibodies and also varieties in them. Thus an affinity function for antibodies fitness adjustment should be applied.

Points with a final degree greater than the cut-off value SM will be put in category small $(S)$. Points with a final degree between the cut-off SM and cut-off ML will be in category medium $(M)$, and other points with final degree less than cut-off value ML will be in category large $(L)$. Here, coefficients of criteria and cut-offs in the segments with length of $K+2$ (with $K$ sub-segment) are located. Sub-segments are coefficients $\left(W_{j}\right)$ and cut-offs $\left(X_{S M}, X_{M L}\right)$. Therefore, $C=\left(W_{1}, W_{2}, W_{3}, \ldots, W_{K}, X_{S M}, X_{M L}\right)$, where $W_{j}$ is the weight of criterion $j$ and $\sum_{j=1}^{k} W_{j}=1, X_{S M}>X_{M L}$

These segments are determined as antibodies of the artificial immune system. In this case, the weight of each criterion can be coded as an exact value in an antibody. The value of each sub-segment is called the allele and it represents the weight of each criterion which is independent of the other alleles. For a known antibody $C$, categorizing consumer $j$ by the weight sum $w s(c, j)$ is determined as Equation (1) (Guvenir and Erel, 1998):

$$
w s(c, j)=\sum_{j=1}^{k} w_{j} \frac{i_{j}-\min j}{\max j-\min j}
$$

where $k$ is the number of criteria, $i_{j}$ is the anthropometric measurement of person $i$ for criterion $j$, 
and $\max j$ and $\min j$ are the maximum and minimum criterion values for all people, respectively. Classification of person $j$ according to the antibody $C$ is presented as Equation (2) (Guvenir and Erel, 1998):

Classification $(c, j)=\left\{\begin{array}{cc}S, & \text { if } \mathrm{X}_{\mathrm{SM}} \leq w s(c, j) \\ M, & \text { if } \mathrm{X}_{\mathrm{ML}} \leq w s(c, j)<X_{S M} \\ L, & \text { otherwise. }\end{array}\right.$

With this clear encoding method, the classification manner uses standard genetic algorithm functions (reproduction, crossover, and mutation) for the antibodies population. Also, the immune system algorithm transfers the best antibodies of each generation to the next generation. The fitness evaluation of an antibody, crossover, and mutation operators, which are used in this method, is described as follows:

\section{Fitness Function}

Antibody fitness in the classification problem shows the ability of a test set's correct classification by that antibody. As a result, each user who is not classified correctly will receive a penalty. Due to the linearity of the class order, it is necessary to separate classification error of class $S$ person to class $M$ from classification error of class $S$ person to class $L$. In this approach, fitness of antibody $C$ is presented as Equations (3)-(4) (Guvenir and Erel, 1998):

$\operatorname{Fitness}(c)=\frac{\sum_{i=1}^{t} p_{i}}{t}$

$p_{i}=\left\{\begin{array}{cc}1, & \text { classification }(i, c)=\operatorname{class}(i) \\ 0.4, & \mid \text { classification }(i, c)-\operatorname{class}(i) \mid=1 \\ 0, & \text { otherwise },\end{array}\right.$

In Equation (3), $t$ is the size of the test set. Equation (4) shows the penalty function of a misclassified person and class $(i)$ is the class of person $i$ which is determined by the expert. If the classification specified for the $i$-th training instance by the expert (i.e., class $(i)$ ) is equal to classification of person $i$ according to antibody $c$ (i.e., classification $(i, c)$ ), the penalty value is equal to 1 . It should be noted that this fitness function prefers the antibody that makes one error from two class differences to the antibody that makes two errors from one class difference. Therefore, when the difference of class $(i)$ and classification $(i, c)$ is 1 , the penalty value is considered to be 0.4 . 


\section{Crossover Operator}

The crossover operator is the most important operator in the artificial immune system algorithm. The crossover operation is conducted for the selected pairs of antibodies (which are randomly selected by the roulette wheel method). The feasibility condition of each antibody is described as follows: the value of each sub-segment must be between 0 and 1 ; the summation of weights should be 1; and the value of cut-off $S M$ is greater than the value of cut-off $M L$. Although the initial population is made in a way that all antibodies are feasible and correct, using the standard crossover operator leads to defective sub-segments. An example is provided in Appendix A.

In this research, a recently developed format of the crossover operator, called the "continuous uniform crossover", is utilized. This operator guarantees the feasibility of the offspring (Guvenir and Erel, 1998). For two parent antibodies $x=\left(x_{1}, x_{2}, \ldots, x_{n}\right)$ and $y=\left(y_{1}, y_{2}, \ldots, y_{n}\right)$, the offspring antibodies are: $x^{\prime}=\left(x_{1}^{\prime}, x_{2}^{\prime}, \ldots, x_{n}^{\prime}\right)$ and $y^{\prime}=\left(y_{1}^{\prime}, y_{2}^{\prime}, \ldots, y_{n}^{\prime}\right)$ in a way that $x_{i}^{\prime}=S x_{i}+(1-S) y_{i}, y_{i}^{\prime}=(1-S) x_{i}+S y_{i}$; where $S$ is a constant for each crossover operator. This operator keeps the sum of any subset of sub-segments. On the basis of classification, if the sum of $m$ sub-segments in an antibody segment is equal to $1\left(\sum_{i=1}^{m} x_{i}=1\right)$, after the crossover operator it would result in Equation (5):

$\sum_{i=1}^{m} x_{i}^{\prime}=s \sum_{i=1}^{m} x_{i}+(1-s) \sum_{i=1}^{m} y_{i}=s+(1-s)=1$

It should be mentioned that Equation (5) is valid for each of the two offspring. In addition, this operator will keep the greater-than relation between sub-segments. That is if $X_{S M}>X_{M L}$ then $X_{S M}^{\prime}>X_{M L}{ }_{M L}$. Hence, the continuous uniform crossover operator keeps antibodies' feasibility for multi criteria SML classification (Guvenir and Erel, 1998). Appendix B provides a more detailed mathematical treatment of the selection of $S$.

\section{Probability Function}

The probability value of the selection antibodies can be computed according to the following procedure (Guvenir and Erel, 1998):

Step 1: Compute the fitness value of each antibody in the population.

Step 2: Compute the value of fitness function for each antibody based on Equations (3) and (4).

Step 3: Compute the selection probability for each antibody according to Equation (6): 
$\operatorname{probability}(c)=\frac{\text { Fitnessvalue }(c)}{\text { Sum }(\text { FitnessValue })}$

\section{Mutation Operator}

The mutation operator randomly alters the value of a sub-segment. After applying the mutation operator, the normalization of the changed antibody is required. The normalization will be done in a similar way as the crossover operator with $S<0$.

\section{Affinity value evaluation using entropy theory}

In this section, entropy theory is applied for the evaluation of the affinity value. In immune algorithms, each candidate solution will be compared with the best-known solution obtained up to that point. This will allow an affinity value to be computed using the entropy theory. The value of the affinity function expresses the similarity between a candidate solution and the bestknown solution obtained up to that point. Mori, Tsukiyama, and Fukuda (1998) defined information entropy $\mathrm{H}(\mathrm{x})$ for a discrete random variable $X$ as shown in Equation (7):

$H(x)=-\sum_{i=1}^{n} p_{i} \cdot \log p_{i}$

where $X=\left\{x_{1}, x_{2}, x_{3}, \ldots, x_{n}\right\}$ is a discrete random variable with probability mass function of $P\left(X=x_{i}\right)=p_{i}, i=1,2, \ldots, n$.

Using information entropy, the similarity of a sequence in relation to a reference sequence can be expressed as Equation (8):

$$
\operatorname{aff}(i)=\frac{1}{1+\frac{1}{k} \sum_{j=1}^{k} h_{i j}}
$$

where $a f f(i)$ is the measure of similarity, $k$ presents the measure of the antibody $(k=$ number of criteria +2$)$, and $h_{i j}=-p_{i j} \log p_{i j}$. If $x_{j}=x_{j} r e f$, then $p_{i j}=1$, and therefore, $h_{i j}=-\log 1=0$. In other words, if $x_{j} \neq x_{j}$ ref, then $p_{i j}=0.5$; this indicates that $h_{i j}=-0.5 \log 0.5=0.151$. It should be noted that two identical sets of numbers will be given an affinity value of 1 . Conversely, two entirely unlike sets of numbers would result in an affinity value equal to: $1 /(1+0.151)$, which is approximately equal to 0.87 .

\subsection{Proposed method}

The steps of the proposed AIA are presented in Figure 2. 
Insert Figure 2 Here

The proposed artificial immune system algorithm used in this research is summarized in the following steps:

Step 1. Initialization: Select values for the number of initial population $(n p)$, the number of generations $(n g)$, the crossover probability $(p c)$, the mutation probability $(p m)$, the affinity threshold (at) and the affinity adjustment $(a a)$. These values are only for initialization of the algorithm and in later stages the values of the parameters will be set. Generate the initial population randomly. Generate feasible antibodies in the initial population randomly.

Step 2. Objective function value evaluation: Compute the fitness of each antibody with the fitness function.

Step 3. Antibody probability assignment: Assign a probability value to each antibody. In the probability assignment, an antibody with the higher degree of fitness will receive greater probability, and this probability value will determine the chance of each antibody to exist in the next generation. In other words, antibodies with greater probability have more opportunity to propagate.

Step 4. Mating pool generation: The best known antibody according to the fitness function will be selected from the mating pool (according to the accelerating mechanism). Then determine the affinity value between each antibody and the best known antibody according to the fitness function. If some antibodies have a similarity probability higher than the predetermined threshold, then decrease the probability value (i.e., the fitness function) that is assigned to them. This work will be done by multiplying an assigned probability to each antibody with expressed conditions by a number less than 1 (i.e., affinity adjustment) and the normalization of the probability values of other antibodies (i.e., restraining mechanism). To expand the mating pool, select ( $n p-1)$ antibodies from the population (including the best antibody) with replacement. The chance of selection of each antibody depends on its fitness value. In other words, antibodies with greater fitness value have more chance to be selected.

Step 5. Crossover operation: Select $(n p \bigotimes 5 p c)$ pairs of mating pool antibodies as parents. Produce offspring with the crossover operator randomly. These antibodies belong to the next generation. 
Step 6. Mutation operation: Select ( $n p \varnothing 5 \mathrm{pm}$ ) pairs of mating pool antibodies as parents. Change one of the bits with the mutation operator randomly. These antibodies belong to the next generation.

Step 7. Replacement: While keeping the best antibody from the previous generation, replace other (np-1) antibodies that are obtained from the operators.

Step 8. Fitness evaluation: Compute the fitness value of each antibody with the fitness function.

Step 9. Termination test: If stopping criteria are achieved, then terminate the algorithm; otherwise, return to Step 3. The termination condition is considered as follows: if the fitness value has not changed in consistency for more than 10 iterations, the algorithm will stop.

The following courses of action are considered in the AIA to overcome the local optimum solutions. First, by using the roulette wheel method, candidate solutions with a higher fitness value have a higher probability to be selected for the production of the next generation of candidate solutions. Second, in order to diversify the search space in the AIA, an accelerating mechanism enhanced with a restraining mechanism is utilized. In the accelerating mechanism, a candidate solution with the best fitness is recorded, which will be used to seed the mating pool. This mechanism ensures that the mating pool contains a large proportion of candidate solutions with good fitness. In addition, in the restraining mechanism, if a candidate solution has a higher affinity value than a prescribed threshold value, then the probability value, obtained from the fitness, is multiplied by a number which is less than one; and the smaller probability value will be assigned to it. This will decrease the selection probability of that solution and prevent overall dominance of a candidate solution. Without the restraining mechanism, a good candidate solution may be pervaded very fast and may be dominated in the mating pool rapidly.

\section{Computational results}

The AIA, explained in the previous section, is coded by the MATLAB programming software to solve the problem. The problem is surveying the classification of consumers into three categories: S, M, and L. Anthropometric measurements of five areas of the hand for designing an ergonomic product are also measured. Thirty-five individuals are classified into three categories: S, M, and L. Each person's left and right hands are measured according to criteria and effective measures in design. Each area is considered as a criterion in the proposed algorithm. These criteria include wrist width, wrist length, palm width, middle finger length, and index finger 
length. They are measured by calipers with accuracy up to 0.01 . The 70 measured samples (both right and left hands) are categorized into the $\mathrm{S}$ group (12 samples), the $\mathrm{M}$ group (38 samples), and the L group (20 samples) based on the expert opinion. The expert opinion is expressed based on their knowledge and expertise using related tools and checklists. After each run of the program the correct proportion is assessed and shown as a percentage. The fitness value is calculated based on Equations (3) and (4). This value shows the ability of a test set's correct classification by that antibody. The probability value is calculated according to Equation (6). This value determines the chance of each antibody to exist in the next generation. The affinity value expresses the similarity between a candidate solution and the best-known solution obtained up to that point. This value can be computed on the basis of Equation (8).

\section{The Artificial Immune Algorithm (AIA) Parameters Tuning}

This section is aimed at studying the behavior of the various operators and parameters of the proposed AIA to achieve better robustness of the algorithm. There are many approaches in the literature to calibrate the algorithm, i.e., parameter tuning of the algorithm; yet, the most commonly practiced method is a full factorial design of experiment.

Many levels for the parameters of the algorithm are considered which are near optimal. Different combinations of the parameter values will constitute a plan (also known as a treatment) for the experiment. The results of applying the algorithm to each of these plans are used to select the appropriate levels that maximize the final solution for the parameters. According to the research by Zandieh et al. (2013), the number of runs should be chosen to be eight. Different levels of these factors are shown in Table 1. In conducting our experiments to test the performance of the algorithm, the number of the initial population $(n p)$ is set to be 100 .

\section{Insert Table 1 Here}

The full factorial design to find the optimal value of the aforementioned five factors, i.e., parameters of the AIA algorithm, requires $3^{4}+4^{1}=324$ experiments. Therefore, 324 combinations of control factors were taken into account. For each trial, eight replications of AIA were performed to yield more reliable information, and then the results of the total fitness are recorded. The fitness value of the total number of generated solutions is averaged in each level. Figure 3 represents these values plotted against each control factor. Based on Figure 3, which shows the fitness of the algorithm, better fitness is acquired when the parameters are set as follows: $p c=0.5, p m=0.1, a t=0.90, a a=0.4$, and $n g=50$. 
Insert Figure 3 Here

Anthropometric measurements of five areas of the hand for designing an ergonomic product are also measured. The person's left and right hands are measured according to the criteria and effective measures in ergonomic product design. Each area is considered as a criterion in our proposed algorithm. Then, the remaining 70 items (35 items for the left hand and 35 items for the right hand) are classified utilizing the weights and cut-off values learned by the AIA into 3 categories S, M, and L. In this experiment, we take advantage of a human expert, who has the full authority and expertise, to classify items based on the mentioned anthropometric data.

The results of our experimental investigation are reported in Table 2. The expert classification did not agree with the result obtained by AIA on 5 items, i.e., based on Table 2, three items in class $S$; and two items in class L. As can be seen, the proposed AIA confirms the expert classification at least in $93 \%$ of the cases.

\section{Insert Table 2 Here}

\section{Conclusion}

Designing ergonomic products has been proven to have an important effect on users' behavior and reaction. In this paper, an AIA was proposed as a meta-heuristic method in order to classify ergonomic products. A case study in this paper, which considers anthropometric measurements for the computer mouse, makes a significant contribution to the ergonomic computer mouse literature. Classification with this method overcomes inefficiency in searching a large space, and results in a higher accuracy in classification ergonomic products regarding anthropometric data compared to the classification of experts. The AIA has a learning mechanism from anthropometric measurements of samples by classifying them into three categories: small (S), medium (M), and large (L), similar to what the PCA and the discriminant analysis techniques do. An expert advisory opinion was also utilized in the case study of this research based on their knowledge and expertise using related tools and checklists. After the learning step, weights for each criterion and cut-off points were determined. Thus, any other samples can be allocated to one of the categories based on the weights and cut-off points obtained from prior steps. To obtain the precise calibration of various operators and parameters of the AIA, a comprehensive comparison by using full factorial design of experiment was performed. To evaluate the effectiveness and robustness of the proposed AIA, it was compared against the expert 
classification outcomes. The comparative results showed that the proposed method confirms the classification of the expert. For future research explorations, other clustering and classification methods, such as K-Means, PCA, or discriminant analysis, can be applied and compared in the case problem for comparison purposes and to provide more reliable outcomes. In addition, the presented approach can be applied to other classification problems such as text categorization (e.g., spam filtering), optical character recognition, and machine vision (e.g., face detection). 


\section{References}

Aghazadeh, F., \& Mital, A. (1987). Injuries due to hand tools. Applied Ergonomics, 18, 273-278.

Guvenir, H.A., \& Erel, E. (1998). Multi criteria inventory classification using a genetic algorithm. European Journal of Operational Research 105(1), 29-37.

Anand, R., Mehrotra, K., Mohan, C.K., \& Ranka, S. (1995). Efficient classification for multiclass problems using modular neural networks. IEEE Transactions on Neural Networks, 6, 117-124.

Bahn, S., Lee, Ch., Nam, Ch., \& Yun, M. (2009). Incorporating Affective Customer Needs for Luxuriousness into Product Design Attributes. Human Factors and Ergonomics in Manufacturing, 19, 105-127.

Belacel, N. (2000). Multicriteria assignment method PROAFTN: Methodology and medical applications. European Journal of Operational Research, 125, 175-183.

Bittner, A.C., Dannhaus, D.M., \& Roth, J.T. (1975). Workplace-accommodated percentage evaluation: model and preliminary results, in M.M.AYOUB and C.G.HALCOMB (Eds) Improved Seat, Console and Workplace Design. Pacific Missile Test Center, Point Mugu, CA 93042.

Brameier, M., \& Banzhaf, W. (2001). A comparison of linear genetic programming and neural networks. IEEE Transactions on Evolutionary Computation, 5 (1), 17-26.

Burgess-Limerick, R., Shemmell, J., Scadden, R., \& Plooy, A. (1999). Wrist posture during computer pointing device use. Clinical Biomechanics, 14(4), 280-286.

Chandrasekar, V., \& Suresh Kumar, S. (2016). A dexterous feature selection artificial immune system algorithm for keystroke dynamics. Stochastic Analysis and Applications, 34(1), 147-154.

Chen, H. H., Chen, C. H., Hoe, Z. Y., \& Yin, Z. X. (2016). Ergonomic Consideration for Wearable Device Design in Frozen Shoulder Rehabilitation. In Advances in Physical Ergonomics and Human Factors (pp. 395-405). Springer International Publishing.

Chen, F., Tang, B., \& Chen, R. (2013). A novel fault diagnosis model for gearbox based on wavelet support vector machine with immune genetic algorithm. Measurement, 46(1), 220-232.

Clancey, W. J. (1984). Classification problem solving (pp. 49-55). Department of Computer Science, Stanford University. 
Cook, C. J., \& Kothiyal, K. (1998). Influence of mouse position on muscular activity in the neck, shoulder and arm in computer users. Applied ergonomics, 29(6), 439-443.

Corcoran, A.L., \& Sen, S. (1994). Using real-valued genetic algorithm to evolve rule sets for classification. In Proceedings of the First IEEE Conference on Evolutionary Computation, Orlando, US, 120-124.

Dasgupta, D. (2002). Guest editorial special issue on artificial immune systems. IEEE Transactions on Evolutionary Computation, 6(3), 225-226.

Dasgupta, D., \& Attoh-Okine, N. (1997, October). Immunity-based systems: A survey. In Systems, Man, and Cybernetics, 1997. IEEE International Conference on Computational Cybernetics and Simulation, 1, 369-37.

Dutka, A. (1995). AMA Handbook of Customer Satisfaction: A Guide to Research, Planning and Implementation. NTC Publishing Group, Illinois.

Falco, I.D., Cioppa, A.D., \& Tarantino, E. (2002). Discovering interesting classification rules with genetic programming. Applied Soft Computing 1, 257-269.

Fernström, E., \& Ericson, M. O. (1997). Computer mouse or Trackpoint—effects on muscular load and operator experience. Applied Ergonomics, 28(5), 347-354.

Frank, S.A., Rose, M.R., Lauder, G.V., (1996). The Design of Natural and Artificial Adaptive Systems. New York: Academic Press.

Fung, B. K. K., Chan, K. Y., Lam, L. Y., Cheung, S. Y., Choy, N. K., Chu, K. W., Chung, L. Y., Liu, W. W., Tai, K. C., Yung, S. Y., Yip, S. L. (2007). Study of wrist posture, loading and repetitive motion as risk factors for developing carpal tunnel syndrome. Hand surgery, 12(01), 13-18.

Garcia, M., \& Garcia-Mendoza, J. (2015). Ergonomic design and analysis for portable assisted mobilization devices. Computer-Aided Design and Applications, 12(sup1), 48-55.

Ghazali, K. H., Mustafa, M. M., Hussain, A., \& Razali, S. (2008). Scale invariant feature transform technique for weed classification in oil palm plantation. Journal of applied sciences, 8(7), 1179-1187.

Gochet, W., Stam, A., Srinivasan, V., \& Chen, S. (1997). Multigroup discriminant analysis using linear programming. Operations Research, 45 (2), 213-225.

Greiner, T. M. (1991). Hand anthropometry of US army personnel (No. TR-92/011). Army natick research development and engineering center MA 
Guan, S.U., \& Li, P. (2003). Feature selection for modular neural network classifiers. Journal of Intelligence System, 12 (3), 113-139.

Hong, J., Lee, W., Lee, S., Lee, B., \& Lee, Y. (2000). An efficient production planning algorithm for multi-head surface mounting machines using the biological immune algorithm. International Journal of Fuzzy Systems, 2(1), 45-53.

Hsiao, H., Whitestone, J., Kau, T. Y., \& Hildreth, B. (2015). Firefighter Hand Anthropometry and Structural Glove Sizing a New Perspective. Human Factors: The Journal of the Human Factors and Ergonomics Society, 57(8), 1359-1377.

Hsu, C.-H. (2009). Developing accurate industrial standards to facilitate production in apparel manufacturing based on anthropometric data. Human Factors and Ergonomics in Manufacturing \& Service Industries, 19, 199-211.

Illias, H. A., \& Chai, X. R. (2016). Hybrid modified evolutionary particle swarm optimisationtime varying acceleration coefficient-artificial neural network for power transformer fault diagnosis. Measurement, 90, 94-102.

Ishibuchi, H., Nakashima, T., \& Murata, T. (1999). Performance evaluation of fuzzy classifier systems for multidimensional pattern classification problems. IEEE Transaction on System. Man and Cybernetics, Part B, 29 (5), 601-618.

Jiao, L.C., \& Wang, L. (2000). A novel genetic algorithm based on immunity. IEEE Transaction on System, Man and Cybernetics, Part A. 30(5), 552-561.

Jung, E., S., Shin, Y., \& Kee, D. (2000). Generation of visual fields for ergonomic design and evaluation. International Journal of Industrial Ergonomics, 26, 445-456.

Kalinli, A., \& Karaboga, D. (2003). IIR filter design using immune algorithm. In IJCI Proceedings of the International Conference on Signal Processing, 1(2), 583-586.

Kalinli, A., \& Karaboga, N. (2005). Artificial immune algorithm for IIR filter design. Engineering Applications of Artificial Intelligence, 18(8), 919-929.

Kar, S.K., Ghosh, S., Manna, I., Banerjee, S., \& Dhara, P. (2003). An investigation of hand anthropometry of agricultural workers. Journal of Human Ecology, 41, 57-62.

Karlqvist, L., Hagberg, M., \& Selin, K. (1994). Variation in upper limb posture and movement during word processing with and without mouse use. Ergonomics, 37(7), 1261-1267.

Keir, P. J., Bach, J. M., \& Rempel, D. (1999). Effects of computer mouse design and task on carpal tunnel pressure. Ergonomics, 42(10), 1350-1360. 
Kieckhoefer, H., Ingleby, M., \& Lucas, G. (2006). Monitoring the physical formation of earprints: Optical and pressure mapping evidence. Measurement, 39(10), 918-935.

Komaki, G. M., Teymourian, E., \& Kayvanfar, V. (2016). Minimising makespan in the twostage assembly hybrid flow shop scheduling problem using artificial immune systems. International Journal of Production Research, 54(4), 963-983.

Kwon, O., Jung, K., You, H., \& Kim, H. E. (2009). Determination of key dimensions for a glove sizing system by analyzing the relationships between hand dimensions. Applied Ergonomics, 40(4), 762-766.

Lacko, D., Vleugels, J., Fransen, E., Huysmans, T., De Bruyne, G., Van Hulle, M. M., Sijbers, J., \& Verwulgen, S. (2017). Ergonomic design of an EEG headset using 3D anthropometry. Applied Ergonomics, 58, 128-136.

Lacković, I., Bilas, V., Šantić, A., \& Nikolić, V. (2000). Measurement of gait parameters from free moving subjects. Measurement, 27(2), 121-131.

Li, X., Zhang, X., Li, C., \& Zhang, L. (2013). Rolling element bearing fault detection using support vector machine with improved ant colony optimization. Measurement, 46(8), 2726-2734.

Liu, C. H., \& Fan, S. C. (2014). Ergonomic design of a computer mouse for clients with wrist splints. American Journal of Occupational Therapy, 68(3), 317-324.

Liu, B. Sh. (2008). Incorporating anthropometry into design of ear-related products. Applied Ergonomics, 39, 115-121.

Lu, B.L., \& Ito, M. (1999). Task decomposition and module combination based on class relations: a modular neural network for pattern classification. IEEE Transactions on Neural Networks, 10 (5), 1244-1256.

Mandahawi, N., Imrhan, Sh., Al-Shobaki, S., \& Sarder, b. (2008). Hand anthropometry survey for the Jordanian population. International Journal of Industrial Ergonomics, 38, 966976.

Martin, J. L., Norris, B. J., Murphy, E., \& Crowe, J. A. (2008). Medical device development: The challenge for ergonomics. Applied Ergonomics, 39(3), 271-283.

Merelo, J.J., Prieto, A., \& Moran, F. (2001). Optimization of classifiers using genetic algorithms, in: M. Patel, V. Honavar, K. Balakrishnan (Eds.), Advances in the Evolutionary Synthesis of Intelligent Agents, Cambridge: MIT Press. 
Michalowski, W., Rubin, S., Slowinski, R., \& Wilk, S. (2001). Triage of the child with abdominal pain: A clinical algorithm for emergency patient management. Paediatrics and Child Health, 6 (1), 23-28.

Miyamoto, A., Nakamura, H., \& Kruszka, L. (2004). Application of the improved immune algorithm to structural design support system. Journal of Structural Engineering, 130(1), 108-119.

Mori, K., Tsukiyama, M., \& Fukuda, T. (1998). Adaptive Scheduling System Inspired by Immune System. Proceeding of the IEEE International Conference on Systems, Man and Cybernetics, San Diego. CA, 3833-3837.

Nagarajan, B., \& Balasubramanie, P. (2008). Neural classifier for object classification with cluttered background using spectral texture based features. Journal of Artificial Intelligence, 1, 61-69.

Nowak, E. (1996). The role of anthropometry in design of work and life environments of the disabled population. International Journal of Industrial Ergonomics, 17, 113-121.

Okunribido, O.O. (2000). A survey of hand anthropometry of female rural farm workers in Ibadan, Western Nigeria. Ergonomics, 43, 282-292.

Pääkkönen, P., \& Pakkala, D. (2015). Reference Architecture and Classification of Technologies, Products and Services for Big Data Systems. Big Data Research, 2, 166-186.

Pekelney, R., \& Chu, R. (1995, October). Design criteria of an ergonomic mouse computer input device. In Proceedings of the Human Factors and Ergonomics Society Annual Meeting (Vol. 39, No. 5, pp. 369-373). SAGE Publications.

Pheasant, S. (2003). Body space, Anthropometry, Ergonomics and the Design of Work. Second edition published in the Taylor \& Francis e-Library.

Rempel, D., Keir, P. J., Smutz, W. P., \& Hargens, A. (1997). Effects of static fingertip loading on carpal tunnel pressure. Journal of Orthopaedic Research, 15(3), 422-426.

Rempel, D., Bach, J. M., Gordon, L., \& So, Y. (1998). Effects of forearm pronation/supination on carpal tunnel pressure. The Journal of hand surgery, 23(1), 38-42.

Rulon, P.J., Tiedeman, D.V., Tatsuoka, M.M., \& Langmuir, C.R. (1967). Multivariate Statistics for Personnel Classification. Wiley, New York.

Schaub, Kh., Landau, K., Menges, R., \& Großmann, K. (1997). A Computer-Aided Tool for Ergonomic Workplace Design and Preventive Health Care. Human Factors and 
Ergonomics in Manufacturing, 7, 269-304.

Setnes, M., \& Roubos, H. (2000). GA-Fuzzy modeling and classification: complexity and performance. IEEE Transactions on Fuzzy Systems, 8 (5), 509-522.

Siskos, Y., Grigoroudis, E., Zopounidis, C., \& Saurais, O. (1998). Measuring customer satisfaction using a survey based preference disaggregation model. Journal of Global Optimization, 12 (2), 175-195.

Souza, S. S., Romero, R., Pereira, J., \& Saraiva, J. T. (2016). Artificial immune algorithm applied to distribution system reconfiguration with variable demand. International Journal of Electrical Power \& Energy Systems, 82, 561-568.

Stefanowski, J., \& Slowinski, R. (1998). Rough set theory and rule induction techniques for discovery of attribute dependencies in medical information systems. Bulletin of the Polish Academy of Sciences, ser. Technical Sciences, 46 (2), 247-263.

Tsumoto, S. (1998). Automated extraction of medical expert system rules from clinical databases based on rough set theory. Information Sciences, 112, 67-84.

Vincent, C. J., Li, Y., \& Blandford, A. (2014). Integration of human factors and ergonomics during medical device design and development: It's all about communication. Applied ergonomics, 45(3), 413-419.

Watkins, A., Timmis, J., \& Boggess, L. (2004). Artificial immune recognition system (AIRS): An immune-inspired supervised learning algorithm. Genetic Programming and Evolvable Machines, 5(3), 291-317.

Wu, F. G., Ma, M. Y., \& Chang, R. H. (2009). A new user-centered design approach: A hair washing assistive device design for users with shoulder mobility restriction. Applied Ergonomics, 40(5), 878-886.

Xiaoping, C., Bo, Q., \& Gang, L. (2003, December). An application of immune algorithm in FIR filter design. In Neural Networks and Signal Processing, 2003. Proceedings of the 2003 International Conference on (Vol. 1, pp. 473-475). IEEE.

Zandieh, M., Farahani, H. F., \& Roshanaei, V. (2013). Multi-criteria inventory classification problem: An effective artificial immune algorithm. International Journal of Management Perspective, $1(1)$.

Zhu, F., \& Guan, S. (2004). Feature selection for modular GA-based classification. Applied Soft Computing, 4, 381-393. 


\section{Appendix A}

\section{Crossover Operator in AIA-Example}

Consider the following two antibodies as parents: $X=(0.1,0.2,0.3 \mid 0.4)$ and $Y=(0.2,0.2,0.1 \mid 0.5)$.

With the single-point classic crossover operator, the following offspring will be produced: $X^{\prime}=(0.1,0.2,0.3,0.5)$ and $Y^{\prime}=(0.2,0.2,0.1,0.4)$; where $\sum_{j=1}^{4} X_{j}^{\prime}$ is equal to 1.1 , which is greater than one, and $\sum_{j=1}^{4} Y_{j}^{\prime}$ is equal to 0.9 , which is less than one. Therefore, $X^{\prime}, Y^{\prime}$ have defective or infeasible weights.

\section{Appendix B}

\section{Continuous Uniform Crossover - Mathematical treatment of the selection of $S$}

The selection of $S$ is critical. If $S=0$, then the offspring will be exactly similar to the parents. If $S>0$, then the sub-segments will be between 0 and 1 . However, sub-segments over production of consecutive generations will be similar to each other. If $S=0.5$, then both offspring will be the same and the values of the sub-segments will be the average of the corresponding subsegments values in the parents. On the other hand, if $S>0$, then the sub-segments will be distant from the corresponding sub-segments in the parents and may go out of the determined limits. For instance, the sub-segment value may be negative or more than 1 , although the sum of subsegments is still equal to 1 . In this case, antibodies should be normalized and for $S<0$, the value of each sub-segment after the crossover operation will be surveyed until it is not below 0 . If a sub-segment with the value greater than 1 exists, certainly a sub-segment with negative value will exist. In the case of a negative sub-segment, at first the minimum sub-segment value is subtracted from all the sub-segments, and then, the value of each sub-segment will be equal to $x_{i}=\frac{x_{i}}{\sum_{j=1}^{k} x_{j}}$; where $k$ is the number of criteria. If $S<0$, it may result that $X_{S M}\left\langle X_{M L}\right.$ and for

resolving this, $X_{S M}$ and $X_{M L}$ are interchanged. 


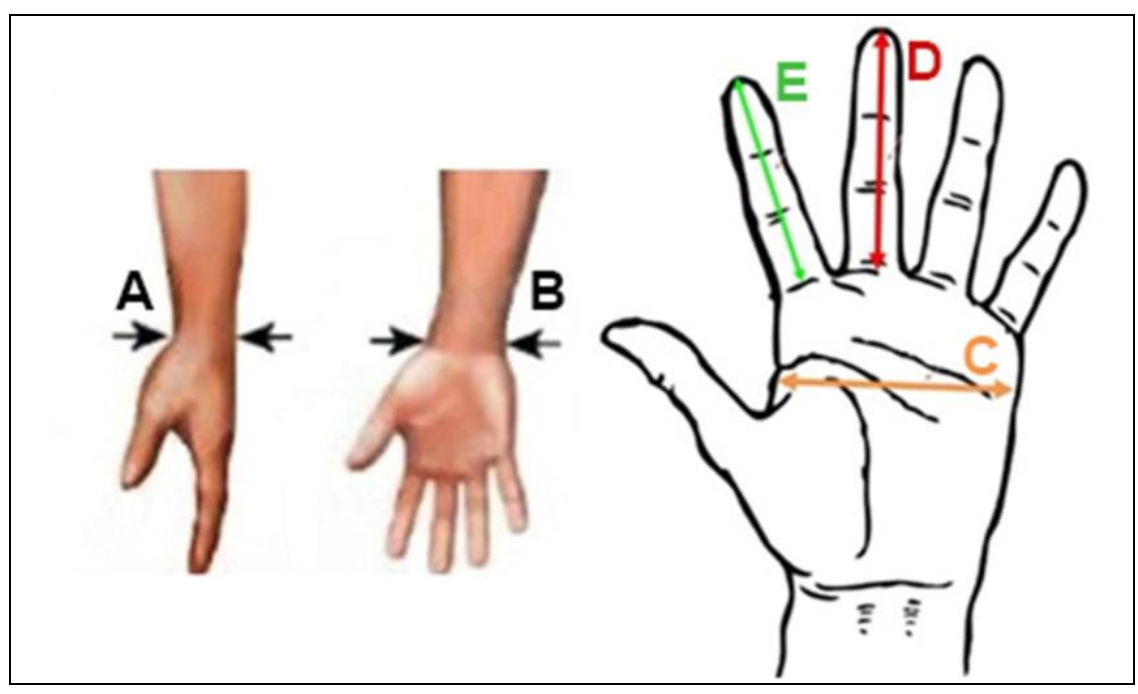

Figure 1. Five anthropometric measurements of hand 


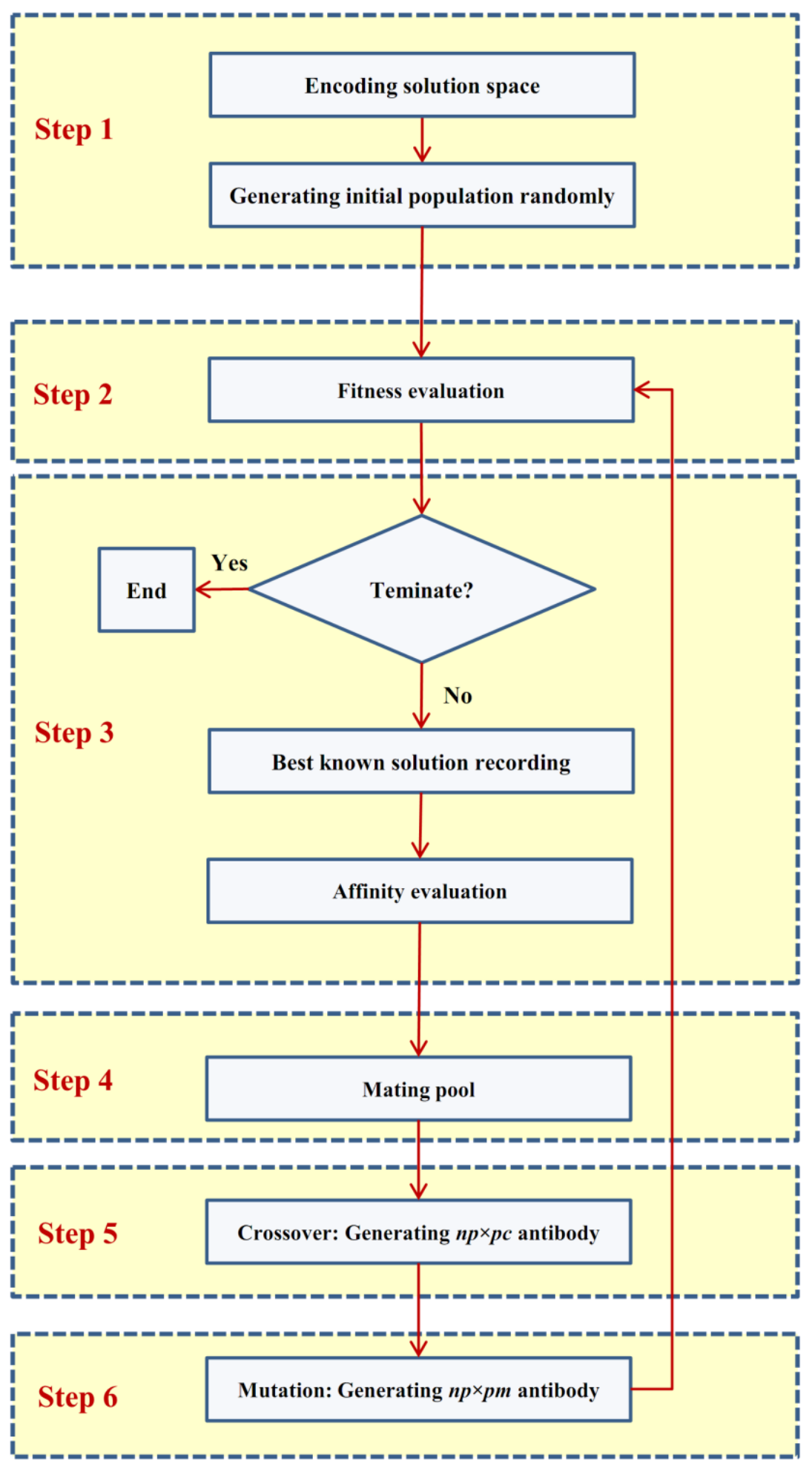

Figure 2. The proposed artificial immune algorithm procedure 


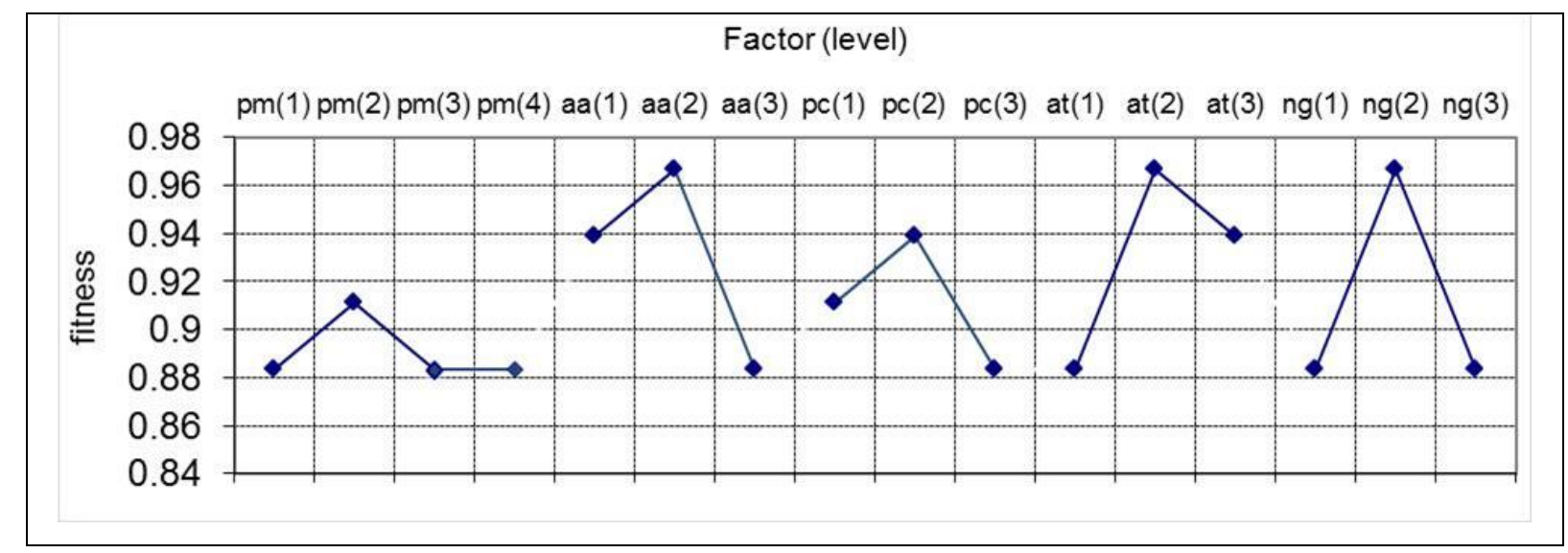

Figure 3. Fitness values of various factors' levels 
Table 1. Various levels of AIA factors

\begin{tabular}{|c|c|c|c|}
\hline Factors & Symbol & Index of level & Levels \\
\hline \multirow{3}{*}{ Crossover Probability } & \multirow{3}{*}{$\mathrm{pc}$} & 1 & 0.40 \\
\hline & & 2 & 0.50 \\
\hline & & 3 & 0.80 \\
\hline \multirow{4}{*}{ Mutation Probability } & \multirow{4}{*}{$\mathrm{pm}$} & 1 & 0.05 \\
\hline & & 2 & 0.10 \\
\hline & & 3 & 0.20 \\
\hline & & 4 & 0.30 \\
\hline \multirow{3}{*}{ Affinity Threshold } & \multirow{3}{*}{ at } & 1 & 0.85 \\
\hline & & 2 & 0.90 \\
\hline & & 3 & 0.95 \\
\hline \multirow{3}{*}{ Affinity Adjustment } & \multirow{3}{*}{ aa } & 1 & 0.50 \\
\hline & & 2 & 0.40 \\
\hline & & 3 & 0.30 \\
\hline \multirow{3}{*}{ Number of Generations } & \multirow{3}{*}{ ng } & 1 & 30 \\
\hline & & 2 & 50 \\
\hline & & 3 & 70 \\
\hline
\end{tabular}


Table 2. Comparison of AIA and the expert classification on the hand measured items

\begin{tabular}{|c|c|c|c|c|}
\hline \multirow{2}{*}{ Class } & \multirow{2}{*}{ Expert } & \multicolumn{3}{|c|}{ AIA } \\
\cline { 3 - 5 } & & S & M & L \\
\hline S & 12 & 9 & 3 & 0 \\
\hline M & 38 & 0 & 38 & 0 \\
\hline L & 20 & 0 & 2 & 18 \\
\hline Total & 70 & 9 & 43 & 18 \\
\hline
\end{tabular}

